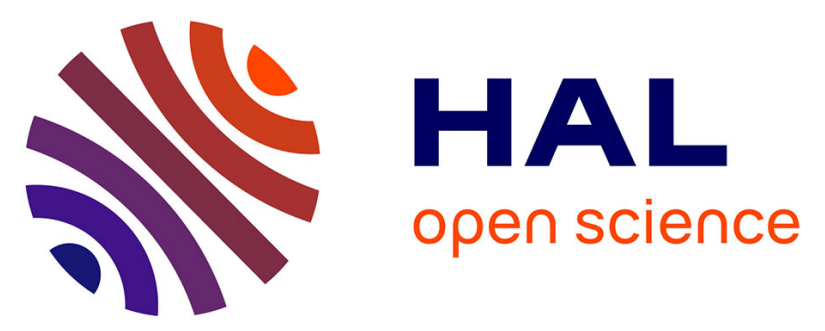

\title{
Association of low-grade inflammation and platelet activation in hypertensive patients with microalbuminuria
}

\author{
Patrizia Ferroni, Maria Teresa Guagnano, Angela Falco, Vincenzo Paoletti, \\ Maria Rosaria Manigrasso, Noemi Michetti, Francesca Santilli, Fiorella \\ Guadagni, Stefania Basili, Giovanni Davì, et al.
}

\section{To cite this version:}

Patrizia Ferroni, Maria Teresa Guagnano, Angela Falco, Vincenzo Paoletti, Maria Rosaria Manigrasso, et al.. Association of low-grade inflammation and platelet activation in hypertensive patients with microalbuminuria. Clinical Science, 2007, 114 (6), pp.449-455. 10.1042/CS20070307 . hal-00479399

\section{HAL Id: hal-00479399 \\ https://hal.science/hal-00479399}

Submitted on 30 Apr 2010

HAL is a multi-disciplinary open access archive for the deposit and dissemination of scientific research documents, whether they are published or not. The documents may come from teaching and research institutions in France or abroad, or from public or private research centers.
L'archive ouverte pluridisciplinaire $\mathbf{H A L}$, est destinée au dépôt et à la diffusion de documents scientifiques de niveau recherche, publiés ou non, émanant des établissements d'enseignement et de recherche français ou étrangers, des laboratoires publics ou privés. 


\section{Association of low-grade inflammation and platelet activation in hypertensive patients} with microalbuminuria.

${ }^{\dagger}$ P. Ferroni, *M.T. Guagnano, ${ }^{*}$ A. Falco, ${ }^{\star}$ V. Paoletti, *M.R. Manigrasso, *N. Michetti, ${ }^{*} \mathrm{~F}$. Santilli, ${ }^{\dagger}$ F. Guadagni, ${ }^{\dagger}$ S. Basili, *G. Davì.

*Center of Excellence on Aging, “G. d'Annunzio" University Foundation and Department of Medicine and Aging, University of Chieti “G. d'Annunzio". "Department of Medical Therapy, University of Rome “La Sapienza", Rome. `Department of Laboratory Medicine \& Advanced Biotechnologies, IRCCS San Raffaele Pisana, Rome, All in Italy.

Key words: Inflammation, hypertension, platelet activation.

Short title: $\quad$ sCD40L in hypertensive subjects with microalbuminuria

\section{Correspondence to:}

Giovanni Davì, MD.

G. D'Annunzio Foundation, University of Chieti, Via Colle dell'Ara, 66013 Chieti, Italy.

Tel. 0039-0871-541312;

Fax: 0039-0871-541313;

E-mail: gdavi@unich.it 


\begin{abstract}
Increased levels of soluble CD40 ligand (sCD40L) have been associated with enhanced in vivo platelet activation, and may represent a molecular link between inflammation and prothrombotic state.

We aimed at analyzing the relationship among platelet activation, endothelial dysfunction low-grade inflammation and SCD40L in hypertensive patients with or without microalbuminuria (MA).
\end{abstract}

A cross-sectional comparison of sCD40L levels was performed in 25 patients with essential hypertension and MA (MH) pair-matched for gender and age with 25 patients with essential hypertension (EH) and 25 healthy normotensive subjects (HS). Circulating Creactive protein (CRP, marker of inflammation), $\mathrm{sP}$-selectin (marker of in vivo platelet activation), asymmetric dimethylarginine (ADMA) and von Willebrand Factor (vWF) (markers of endothelial dysfunction) levels were analyzed in each subject.

sCD40L levels were increased in MH patients compared to either $\mathrm{EH}(\mathrm{p}<0.001)$ or HS $(\mathrm{p}<0.0001)$. A highly significant correlation between plasma sCD40L and sP-selectin $(p<0.0001)$, vWF $(p<0.001)$ or CRP levels $(p<0.05)$ was observed in MH patients.

Multivariate regression analysis showed that $\mathrm{sP}$-selectin was the strongest independent predictor of sCD40L levels $(\mathrm{p}<0.0001)$ in $\mathrm{MH}$ patients. Hypertensive patients with both vWF and CRP levels above the median had the highest sCD40L levels $(\mathrm{p}<0.0001)$. Factorial ANOVA analysis of all hypertensive subjects confirmed that only MH patients with lowgrade inflammation had elevated levels of sCD40L.

sCD40L levels appear to discriminate a subset of patients characterized by microalbuminuria and low-grade inflammation, suggesting that inhibition of the CD40/CD40L system may represent a potential therapeutic target in hypertensive subjects at high risk for cardiovascular events. 


\section{INTRODUCTION}

The CD40 ligand (CD40L) is a transmembrane protein structurally related to tumor necrosis factor- $\alpha($ TNF- $\alpha)$ that has been first identified on CD4+ T cells $[1,2]$. Subsequently, CD40L has been identified on the surface membrane of activated platelets [3] from where a soluble form (sCD40L) can be released into the circulation [4]. Both membrane-bound and sCD40L interact with CD40 expressed on vascular cells, resulting in inflammatory and prothrombotic responses [3, 5]. Engagement of CD40L with CD40 on endothelial cells, in fact, results in phenotypic changes that are similar to those induced by TNF- $\alpha$, i.e., increased expression of adhesion molecules and secretion of inflammatory cytokines [6]. In addition, sCD40L can act as a ligand of the platelet fibrinogen receptor glycoprotein IIb/IIIa and is involved in thrombus stabilization and platelet activation [7]. Thus, sCD40L may represent a key molecule linking inflammation and prothrombotic state in human diseases.

To date, it is estimated that more than $95 \%$ of plasma SCD40L is derived from platelets [8]. Accordingly, increased levels of SCD40L have been associated with enhanced in vivo platelet activation in various clinical conditions [9-15]. Meanwhile, it has become evident that $\mathrm{SCD} 40 \mathrm{~L}$ could be regarded as a predictive marker of cardiovascular events in healthy individuals $[15,16]$, which may not only indicate active inflammation and platelet activation, but may also exhibit direct prothrombotic properties that mediate early atherogenesis, plaque rupture, and thrombosis [17].

Persistent platelet activation has been demonstrated in patients with essential hypertension, especially those with advanced vascular lesions [18-21], and tight blood pressure control may result in significant improvements in platelet function in hypertensive subjects at high risk of cardiovascular events [22]. In addition, low-grade inflammation (as evidenced by increased levels of C-reactive protein [CRP]) [23, 24] and endothelial dysfunction (as evidenced by increased levels of von Willebrand factor [vWF] or asymmetric 
dimethylarginine [ADMA])[24-27] have been frequently associated with essential hypertension and may help identifying patients at very high cardiovascular risk.

Although, several factors link platelet activation, endothelial dysfunction and low-grade inflammation with sCD40L, no information is available yet about their interrelationships with hypertension-related cardiovascular and metabolic abnormalities. For this reason, we addressed that issue in hypertensive patients with or without microalbuminuria (MA), which is currently acknowledged as a predictor of vascular disease [28], and the strongest independent determinant of ischaemic heart disease among non-diabetic individuals with arterial hypertension $[29,30]$. 


\section{METHODS}

\section{Design of the study}

A cross-sectional comparison was performed in 50 patients with essential hypertension, with $(\mathrm{MH})$ and without $(\mathrm{EH})$ microalbuminuria, and in 25 healthy normotensive subjects (HS) enrolled at our Institutions (Table 1). Hypertensive patients with MA ( $n=25)$ were pairmatched for gender and age with hypertensive patients without MA ( $\mathrm{n}=25)$ and HS (Table 1).

Exclusion criteria were represented by a history or evidence of atherothrombotic diseases, diabetes mellitus, cigarette smoking, dyslipidemia, obesity and metabolic syndrome in order to avoid confounding by other determinants of endothelial dysfunction and platelet activation. None of the subjects was permitted to take vitamin supplements, nonsteroidal antiinflammatory drugs, or antiplatelet agents for at least 30 days before the study. Women who were taking hormonal replacement therapy were also excluded.

Essential hypertension was clinically diagnosed according to the WHO criteria [31], on 3 consecutive occasions and on the basis of negative results of investigation for secondary hypertension that included hormonal evaluation, renal scintigraphy and/or echo-doppler scan of the renal arteries. MA was defined as a urinary albumin excretion rate (AER) between 20 and $200 \mu \mathrm{g} / \mathrm{min}$. Hypertensive patients were under long-term treatment with antihypertensive medication (angiotensin converting enzyme inhibitors and/or diuretics and/or, calcium antagonists). Both groups of hypertensive subjects received a standard diet containing $\sim 150$ mmol of sodium per day. The mean duration of hypertension was defined as the time between the diagnosis and inclusion in the present study.

All subjects were studied on an outpatient basis. Blood samples were obtained in the early morning after a 12-hour fast. 
The study was performed under the appropriate institutional ethics approvals and in accordance with the principles embodied in the Declaration of Helsinki. Informed consent was obtained from each participating subject.

\section{Biochemical analyses}

The mean AER was calculated from three consecutive timed overnight urine collections.

AER was measured by nephelometry (Roche Diagnostics, France). Serum C-reactive protein (CRP) levels were measured with a highly sensitive immunoassay [32]. Total cholesterol, triglycerides, HDL-cholesterol and LDL-cholesterol concentrations were measured as previously described [18].

Plasma sP-selectin, sCD40L levels (both by R\&D Systems, Minneapolis, MN, USA) and vWF levels (Imubind vWF ELISA, American Diagnostica) were measured in citrated plasma samples by commercially available enzyme-immunoassays according to the manufacturers' instructions. ADMA levels were measured in plasma samples by a commercially available enzymeimmunometric assay (DLD diagnostika, Hamburg, Germany), previously validated by comparison with gas chromatography/mass spectrometry [33]. Plasma samples were processed promptly after collection (within $2 \mathrm{~h}$ ) to minimize ex-vivo release of P-selectin and CD40L from platelets [34].

\section{Statistical Analysis}

Statistical analysis was performed by chi-square statistics, Pearson's correlation coefficient and by one-way analysis of variance with the Bonferroni adjustment to assess differences among the groups (hypertensive with and without microalbuminuria and non hypertensive subjects). When necessary appropriate non-parametric tests were employed (Spearman's correlation coefficient, Kruskall-Wallis method and Mann-Whitney U test). Multiple linear regression analysis and factorial ANOVA analysis were performed to further analyze the data. 
Multiple regression analysis always included, as the independent variables, male gender, age, body mass index (BMI), AER, disease duration, antihypertensive treatment, systolic and diastolic blood pressure, blood lipids and smoking habit. With 25 subjects recruited in the cross-sectional comparison, the study had a $95 \%$ power to detect a $40 \%$ difference in SCD $40 \mathrm{~L}$ serum levels between the hypertensive microalbuminuric patients and control groups with a two-tailed $\alpha$ of 0.05. Data are presented as mean (1 SD) or median and interquartile range (IQR; 25th , 75th percentile). Only $\mathrm{p}$ values lower than 0.05 were regarded as statistically significant. All tests were two-tailed and analyses were performed using a computer software package (Statistica 6.0, StatSoft Inc., Tulsa, OK or Statistical Package for the Social Sciences, version 13.0, SPSS Inc., and Chicago, IL). 


\section{RESULTS}

Baseline characteristics of the three groups of subjects are summarized in Table 1. As shown, hypertensive patients with or without MA did not differ in terms of blood pressure, disease duration, creatinine serum level and serum lipid pattern. Healthy subjects had similar clinical and laboratory characteristics as compared to hypertensive subjects. Urinary AER was significantly higher in hypertensive patients with MA compared to hypertensive patients without MA and healthy subjects ( $\mathrm{p}<0.0001)$; normoalbuminuric hypertensive subjects had significantly higher values of urinary AER than controls $(\mathrm{p}<0.0001)$

Platelet activation, as reflected by sP-selectin levels, was significantly enhanced in microalbuminuric hypertensive patients as compared to either HS or EH patients (Table 2). Furthermore, an impairment of endothelial function was found in hypertensive patients, as evidenced by increased plasma levels of both $\mathrm{WWF}$ and ADMA in hypertensive subjects with or without MA compared with HS (Table 2). Elevated ADMA (regression coefficient $=0.259$, $\mathrm{p}=0.01)$ or $\mathrm{vWF}$ (regression coefficient $=0.595, \mathrm{p}<0.0001$ ) levels, low HDL-cholesterol (regression coefficient $=-0.305, p=0.001$ ) and disease duration (regression coefficient $=0.244$, $\mathrm{p}=0.004)$ were all predictive of MA by multivariate analysis.

Plasma sCD40L levels were significantly increased in MH patients compared to either EH or HS (Table 2), but no differences could be observed between the two latter groups (Figure 1). A highly significant correlation between plasma sCD40L and sP-selectin $(\mathrm{Rho}=$ $0.750, \mathrm{p}<0.0001), \mathrm{vWF}(\mathrm{Rho}=0.658, \mathrm{p}<0.0001)$ or CRP levels $(\mathrm{Rho}=0.42, \mathrm{p}<0.003)$ was observed in hypertensive patients. Subgroup analysis demonstrated that these associations were retained in the subgroup of MH patients (Figure 2) but not in EH patients, in whom only the correlation between $\mathrm{sCD} 40 \mathrm{~L}$ and $\mathrm{sP}$-selectin $(\mathrm{Rho}=0.528, \mathrm{p}<0.007)$ was retained.

Stepwise multivariate regression analysis showed that $\mathrm{sP}$-selectin was the strongest 
independent predictor of sCD40L levels (regression coefficient $[\mathrm{SE}]=0.787[0.13]$; $\mathrm{p}<0.0001$ ) in $\mathrm{MH}$ patients.

To further evaluate the relationship between $\mathrm{SCD} 40 \mathrm{~L}$ and endothelial dysfunction or inflammatory status, hypertensive patients were stratified on the basis of the observed median CRP $(1.22 \mathrm{mg} / \mathrm{dl})$ or vWF (1120 IU/dl) levels. As shown in Figure 3, hypertensive patients with both vWF and CRP levels above the median had the highest sCD40L levels (median level $2.4 \mathrm{pg} / \mathrm{ml})$ compared to patients with either vWF or CRP levels above the median $(1.1$ $\mathrm{pg} / \mathrm{ml})$ or patients with both variables below the median $(0.65 \mathrm{pg} / \mathrm{ml}, \mathrm{H}=21.5, \mathrm{p}<0.0001)$. These results were confirmed in the subset of $\mathrm{MH}(\mathrm{H}=10.7, \mathrm{p}<0.005)$, but not in EH patients.

Based on these results, we finally performed a factorial ANOVA analysis of all hypertensive patients to better characterize the interactions between SCD40L and the inflammatory status in the presence or absence of MA. The results are illustrated in Figure 4, showing that only MH patients with low-grade inflammation had elevated levels of sCD40L. Multivariate regression analysis of this restricted subset of patients confirmed the independent prognostic role of sP-selectin in determining SCD40L levels (regression coefficient $[\mathrm{SE}]=0.838[0.15] ; \mathrm{p}<0.0001)$. 


\section{DISCUSSION}

To date, no clinical evidence is available linking platelet activation and/or inflammation to $\mathrm{SCD} 40 \mathrm{~L}$ production in hypertension. The present study is the first to demonstrate that increased levels of this cytokine may be at least in part related to the occurrence of in vivo platelet activation, as demonstrated by the significant association found between SCD40L and sP-selectin, a well recognized marker of in vivo platelet activation [35-37].

The occurrence of elevated sCD40L levels in patients with hypertension is still a debated issue. In fact, Tsioufis et al. recently reported that MA is not accompanied by an increase of sCD40L concentrations in essential hypertensive subjects and hypothesized an activation of different inflammatory pathways in the progression of renal and cardiovascular atherosclerotic disease in these patients [38]. However, the study group used in this study was restricted to non-smoker hypertensive subjects without other risk factors (i.e. obesity). As Bautista pointed out in his critique, although selection is sometimes used to prevent confounding, it limits generalizability and is rarely justifiable, as confounding can be controlled efficiently using multiple regression analysis [39]. In our cohort of patients, sCD40L levels were higher in MH compared to both hypertensive subjects without MA or age- and gender-matched normotensive subjects. This finding is in agreement with those by other authors suggesting that plasma sCD40L levels may contribute to the increased susceptibility of hypertensive patients to develop vascular damage [40] probably through blunted angiogenesis [41]. In particular, Patel et al. suggested that SCD40L might reflect abnormal platelet activation and, thus, increased sCD40L (as a marker of CD40 ligation) may contribute to the increased cardiovascular risk in patients with hypertension [41].

In the present study, the findings of increased sCD40L levels were limited to a subset of hypertensive patients with MA. The latter significantly correlated with the duration of hypertension, which is in agreement with the findings of other authors [28]. Moreover, we 
showed that increased plasma levels of vWF and ADMA (two well recognized markers of endothelial dysfunction) were both predictive of MA. These findings are confirmatory of previous studies (24-28) and are in agreement with those by Pedrinelli et al., who demonstrated the presence of increased vWF levels in patients with essential hypertension and MA as well as intra-individual correlation between the two variables, suggesting that a dysfunctional glomerular endothelium may cause reduced renal function [25]. Along with the occurrence of endothelial dysfunction (as evidenced by increased levels of both vWF and ADMA), we observed a low-grade inflammatory condition in all hypertensive patients either with MA or without MA. Indeed, circulating CRP levels were significantly higher in MH than EH patients or healthy subjects. This is not surprising if we consider a recent report by Sesso et al. [23] demonstrating that CRP levels are associated with future development of hypertension, which suggests that hypertension is in part an inflammatory disorder.

The major conclusion that may be drawn from this study is that $\mathrm{SCD} 40 \mathrm{~L}$ levels appear to discriminate a high-risk subset of patients characterized by microalbuminuria and low-grade inflammation. This is of particular interest since several population-based studies have shown an association between MA and cardiovascular morbidity and mortality [28], leading to the demonstration that MA is the strongest independent determinant of ischaemic heart disease among subjects with arterial hypertension [29]. Furthermore, MA accompanied by evidence of subclinical inflammation has been proposed as a strong correlate of metabolic abnormalities in essential hypertension and might identify a patient subset at very high cardiovascular risk. In contrast, isolated microalbuminuria may represent a distinct pathophysiological condition characterized by a more benign profile and possibly a better prognosis [24]. In this context, the finding of enhanced levels of sCD40L mainly in a subgroup of $\mathrm{MH}$ patients with low-grade inflammation (as evidenced by elevated CRP 
concentrations) suggests that this cytokine might be directly responsible for prothrombotic properties that mediate thrombosis and acute vascular events.

One limitation to the study might be represented by the small number of patients. However, we must consider that the population enrolled was highly selected, since we excluded hypertensive patients with concomitant risk factors for atherothrombosis, such as obesity, cigarette smoking, diabetes mellitus and metabolic syndrome all of which have been shown to affect significantly the levels of urinary albumin excretion and to be associated with biochemical evidence of in vivo platelet activation [9-13, 15, 42, 43]. In terms of study limitation, the current findings were limited to hypertensive patients with microalbuminuria, a surrogate of the subclinical atherosclerotic burden and low-grade inflammation limiting the generalization of the study. Nevertheless, this study may provide new insight into the pathophysiologic mechanisms linking early renal impairment with elevated cardiovascular risk.

In conclusion, these data confirm that CD40L represents a key molecule linking inflammation and thrombosis. Thus, inhibition of the CD40/CD40L system may represent a potential therapeutic target in a subgroup of hypertensive patients at high risk for cardiovascular events. 


\section{REFERENCES}

1. Mach, F., Schonbeck, U., Sukhova, G.K., et al. (1997) Functional CD40 ligand is expressed on human vascular endothelial cells, smooth muscle cells, and macrophages: implication for CD40-CD40 ligand signaling in atherosclerosis. Proc. Natl. Acad. Sci. USA. 94, 1931-1936.

2. van Kooten, C. (2000) Immune regulation by CD40-CD40L interactions. Front. Biosci. 5, 680-693.

3. Henn, V., Slupsky, J.R., Grafe, M., et al. (1998) CD40 ligand on activated platelets triggers an inflammatory reaction of endothelial cells. Nature. 391, 591-594.

4. Aukrust, P., Muller, F., Ueland, T., et al. (1999) Enhanced levels of soluble and membrane-bound CD40 ligand in patients with unstable angina. Possible reflection of $\mathrm{T}$ lymphocyte and platelet involvement in the pathogenesis of acute coronary syndromes. Circulation. 100, 614-620.

5. Lindmark, E., Tenno, T., Siegbahn, A. (2000) Role of platelet P-selectin and CD40 ligand in the induction of monocytic tissue factor expression. Arterioscler. Thromb. Vasc. Biol. 20, 2322-2328.

6. Granger, D.N., Vowinkel, T., Petnehazy, T. (2004) Modulation of the inflammatory response in cardiovascular disease. Hypertension. 43, 924-931.

7. Prasad, K.S., Andre, P., Yan, Y., Phillips, D.R. (2003) The platelet CD40L/GP IIb-IIIa axis in atherothrombotic disease. Curr. Opin. Hematol. 10, 356-361.

8. Freedman, J.E. (2003) CD40-CD40L and platelet function. Beyond hemostasis. Circulation Res. 92, 944-946.

9. Cipollone, F., Mezzetti, A., Porreca, E., et al. (2002) Association between enhanced soluble CD40L and prothrombotic state in hypercholesterolemia: effects of statin therapy. Circulation. 106, 399-402. 
10. Garlichs, C.D., Kozina, S., Fateh-Moghadam, S., et al. (2003) Upregulation of CD40CD40 ligand (CD154) in patients with acute cerebral ischemia. Stroke. 34, 1412-1418.

11. Danese, S., Katz, J.A., Saibeni, S., et al. (2003) Activated platelets are the source of elevated levels of soluble CD40 ligand in the circulation of inflammatory bowel disease patients. Gut. 52,1435-1441.

12. Lim, H.S., Blann, A.D., Lip, G.Y. (2004) Soluble CD40 ligand, soluble P-selectin, interleukin-6, and tissue factor in diabetes mellitus: relationships to cardiovascular disease and risk factor intervention. Circulation. 109, 2524-2528.

13. Falco, A., Romano, M., Iapichino, L., Collura, M., Davi, G. (2004) Increased soluble CD40 ligand levels in cystic fibrosis. J. Thromb. Haemost. 2, 557-560.

14. Roselli, M., Mineo, T.C., Basili, S., et al. (2004) Soluble CD40 ligand plasma levels in lung cancer. Clin. Cancer Res. 10, 610-614.

15. Novo, S., Basili, S., Tantillo, R., et al. (2005) Soluble CD40L and Cardiovascular Risk in Asymptomatic Low-Grade Carotid Stenosis. Stroke. 36, 673-675.

16. Schonbeck, U., Varo, N., Libby, P., Buring, J., Ridker, P.M. (2001) Soluble CD40L and cardiovascular risk in women. Circulation. 104, 2266-2268.

17. Mo, V.Y., De Lemos, J.A. (2004) Individualizing therapy in acute coronary syndromes: using a multiple biomarker approach for diagnosis, risk stratification, and guidance of therapy. Curr. Cardiol. Rep. 6, 273-278.

18. Davì, G., Gresele, P., Violi, F., et al. (1997) Diabetes mellitus, hypercholesterolemia, and hypertension but not vascular disease per se are associated with persistent platelet activation in vivo. Evidence derived from the study of peripheral arterial disease.

Circulation. 96, 69-75.

19. Spencer, C.G., Gurney, D., Blann, A.D., Beevers, D.G., Lip, G.Y. (2002) Von Willebrand factor, soluble P-selectin, and target organ damage in hypertension: a 
substudy of the Anglo-Scandinavian Cardiac Outcomes Trial (ASCOT). Hypertension. 40, 61-66.

20. Minuz, P., Patrignani, P., Gaino, S., et al. (2004) Determinants of platelet activation in human essential hypertension. Hypertension. 43, 64-70.

21. Nadar, S.K., Blann, A.D., Kamath, S., Beevers, D.G., Lip, G.Y.H. (2004) Platelet indexes in relation to target organ damage in high-risk hypertensive patients: a substudy of the Anglo-Scandinavian Cardiac Outcomes Trial (ASCOT). J. Am. Coll. Cardiol. 44, 415-422.

22. Blann, A.D., Nadar, S., Lip, G.Y. (2003) Pharmacological modulation of platelet function in hypertension. Hypertension. 42, 1-7.

23. Sesso, H.D., Buring, J.E., Rifai, N., Blake, G.J., Gaziano, J.M., Ridker, P.M. (2003) CReactive protein and the risk of developing hypertension. J. Am. Med. Assoc. 290, $2945-2951$.

24. Pedrinelli, R., Dell'Omo, G., Di Bello, V., et al. (2004) Low-Grade inflammation and microalbuminuria in hypertension. Arterioscler. Thromb. Vasc. Biol. 24, 2414-2419.

25. Pedrinelli, R., Giampietro, O., Carmassi, F., et al. (1994) Microalbuminuria and endothelial dysfunction in essential hypertension. Lancet. 344, 14-18.

26. Taddei, S., Salvetti, A. (2002) Endothelial dysfunction in essential hypertension: clinical implications. J. Hypertens. 20, 1671-1674.

27. Goonasekera, C.D., Shah, V., Rees, D.D., Dillon, M.J. (2000) Vascular endothelial cell activation associated with increased plasma asymmetric dimethyl arginine in children and young adults with hypertension: a basis for atheroma? Blood Press. 9, 16-21.

28. Garg, J.P., Bakris, G.L. (2002) Microalbuminuria: marker of vascular dysfunction, risk factor for cardiovascular disease. Vasc. Med. 7, 35-43. 
29. Jensen, J.S., Feldt-Rasmussen, B., Strandgaard, S., Schroll, M., Borch-Johnsen, K. (2000) Arterial hypertension, microalbuminuria, and risk of ischemic heart disease. Hypertension. 35, 898-903.

30. Jager, A., Kostense, P.J., Ruhe, H.G., et al. (1999) Microalbuminuria and peripheral arterial disease are independent predictors of cardiovascular and all-cause mortality, especially among hypertensive subjects: five-year follow-up of the Hoorn Study. Arterioscler. Thromb. Vasc. Biol. 19, 617-624.

31. 1999 World Health Organization-International Society of Hypertension guidelines for the management of hypertension. Guidelines Subcommittee. (1999) J. Hypertens. 17, 151-183.

32. Ledue, T.B., Weiner, D.L., Sipe, J.D., Poulin, S.E,, Collins, M.F., Rifai, N. (1998) Analytical evaluation of particle-enhanced immunonephelometric assays for C-reactive protein, serum amyloid A and mannose-binding protein in human serum. Ann. Clin. Biochem. 35, 745-753.

33. Schulze, F., Wesemann, R., Schwedhelm, E., et al. (2004) Determination of asymmetric dimethylarginine (ADMA) using a novel ELISA assay. Clin. Chem. Lab. Med. 42, 1377-1383.

34. Thom, J., Gilmore, G., Yi, Q., Hankey, G.J., Eikelboom, J.W. (2004) Measurement of soluble P-selectin and soluble CD40 ligand in serum and plasma. J. Thromb. Haemost. 2, 2067-2069.

35. Blann, A.D., Nadar, S.K., Lip, G.Y. (2003) The adhesion molecule P-selectin and cardiovascular disease. Eur. Heart J. 24, 2166-2179.

36. Davì, G., Romano, M,. Mezzetti, A., et al. (1998) Increased levels of soluble P-selectin in hypercholesterolemic patients. Circulation. 97, 953-957.

37. Andre, P. (2004) P-selectin in haemostasis. Br. J. Haermatol. 126, 298-306. 
38. Tsioufis, C., Dimitriadis, K., Taxiarchou, E., et al. (2006) Diverse associations of microalbuminuria with C-reactive protein, interleukin-18 and soluble CD40 ligand in male essential hypertensive subjects. Am. J. Hypertens. 19, 462-466.

39. Bautista, L.E. (2006) Associations of microalbuminuria with inflammation markers in hypertensive men. Am. J. Hypertens. 19, 467.

40. Desideri, G., Cipollone, F., Valeri, L., et al. (2007) Enhanced plasma soluble CD40 ligand levels in essential hypertensive patients with blunted nocturnal blood pressure decrease. Am. J. Hypertens. 20, 70-76.

41. Patel, J.V., Lim, H.S., Nadar, S., Tayebjee, M., Hughes, E.A., Lip, G.Y. (2006)

Abnormal soluble CD40 ligand and C-reactive protein concentrations in hypertension: relationship to indices of angiogenesis. J. Hypertens. 24, 117-121.

42. Lee W.L., Lee W.J., Chen Y.T., et al. (2006) The presence of metabolic syndrome is independently associated with elevated serum CD40 ligand and disease severity in patients with symptomatic coronary artery disease. Metabolism. 55, 1029-1034.

43. Angelico F., Alessandri C., Ferro D., et al. Enhanced soluble CD40L in patients with the metabolic syndrome: Relationship with in vivo thrombin generation. Diabetologia. $49,1169-1174$. 
Table 1: Baseline characteristics of hypertensive patients and healthy subjects.

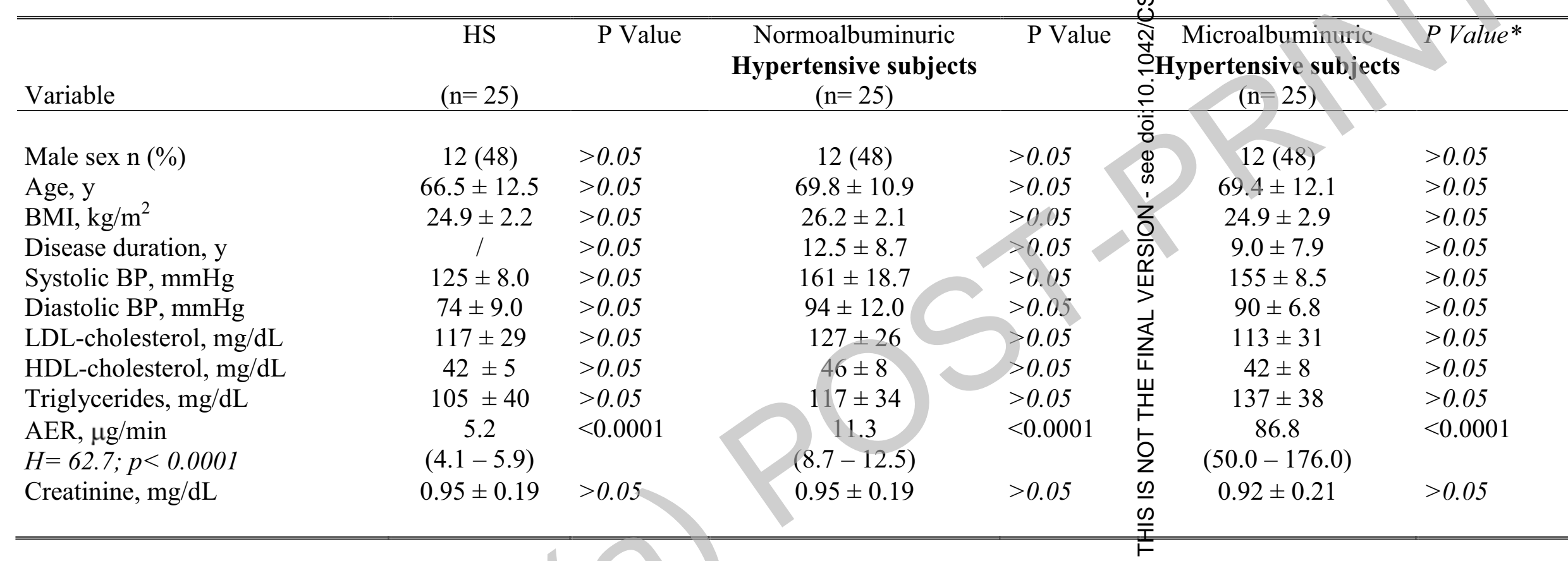

BMI: body mass index; BP: blood pressure; AER: albumin excretion rate; IQR: interquartile range. *Microalbuminuric Hypertensive subjects vs. HS. Data are presented as mean \pm SD or median $(25$ th -75 th percentile). 
Table 2: $\quad$ Laboratory variables of hypertensive patients and healthy subjects.

\begin{tabular}{|c|c|c|c|c|c|c|}
\hline Variable & $\begin{array}{c}\text { HS } \\
(n=25)\end{array}$ & $P$ Value & $\begin{array}{c}\text { Normoalbuminuric } \\
\text { Hypertensive subjects } \\
(\mathrm{n}=25)\end{array}$ & $\begin{aligned} P \text { Value } & \stackrel{\text { Y }}{0} \\
& \vdots \\
\vdots & \vdots\end{aligned}$ & $\begin{array}{c}\text { Microalbuminuric } \\
\text { Hypertensive subjects } \\
(n=25)\end{array}$ & PValue* \\
\hline $\begin{array}{l}\text { sP-selectin, ng/ml } \\
H=34.0, p<0.0001\end{array}$ & $\begin{array}{c}68 \\
(60-75)\end{array}$ & $<0.01$ & $\begin{array}{c}86 \\
(65-98)\end{array}$ & & $\begin{array}{c}136 \\
(122-176)\end{array}$ & $<0.0001$ \\
\hline $\begin{array}{l}\mathrm{ADMA}, \mu \mathrm{mol} / \mathrm{L} \\
H=38.6, p<0.0001\end{array}$ & $\begin{array}{c}0.39 \\
(0.34-0.56)\end{array}$ & $<0.001$ & $\frac{0.56}{(0.46-0.75)}$ & $<0.000$ 度 & $\begin{array}{c}1.12 \\
(0.76-1.32)\end{array}$ & $<0.0001$ \\
\hline $\begin{array}{l}\text { Von Willebrand Factor, IU/dl } \\
F=85.4, p<0.0001\end{array}$ & $728 \pm 133$ & 005 & $892 \pm 148$ & $<0.001 \vdash$ & $1447 \pm 292$ & $<0.001$ \\
\hline
\end{tabular}

HS: healthy subjects; Mann-Whitney U test of healthy subjects vs. hypertensive with MA: ${ }^{*} \mathrm{p}<0.0001 ;{ }^{\dagger} \mathrm{p}<0.001$. ADMA: asymmetrical dimethylarginine. Data are presented as mean \pm SD or median $(25$ th -75 th percentile) 


\section{FIGURE LEGENDS}

Figure 1 Plasma sCD40L levels in hypertensive patients with (MH) or without microalbuminuria (EH) compared to healthy subjects (HS). Solid lines indicate median values; whiskers indicate non-outlier range; columns indicate $25^{\text {th }}-75^{\text {th }}$ percentiles.

Figure 2 Correlation analysis of plasma sCD40L levels and vWF (Panel A, Rho= 0.662, $\mathrm{p}<0.001$ ), sP-selectin (Panel B, Rho $=0.750, \mathrm{p}<0.0001$ ) or $\mathrm{C}$-reactive protein (Panel C, Rho $=0.419, \mathrm{p}<0.05)$ in hypertensive patients with microalbuminuria.

Figure 3 Box-plot analysis of plasma sCD40L levels in all hypertensive patients $(n=50)$ with vWF and/or CRP levels below (negative) or above (positive) the median values.

Figure 4 Factorial ANOVA analysis of plasma sCD40L levels in patients with (MH, $\mathrm{n}=25)$ or without $(\mathrm{EH}, \mathrm{n}=25)$ microalbuminuria stratified on the basis of CRP levels below (negative) or above (positive) the median value observed in hypertensive patients. 
Figure 1

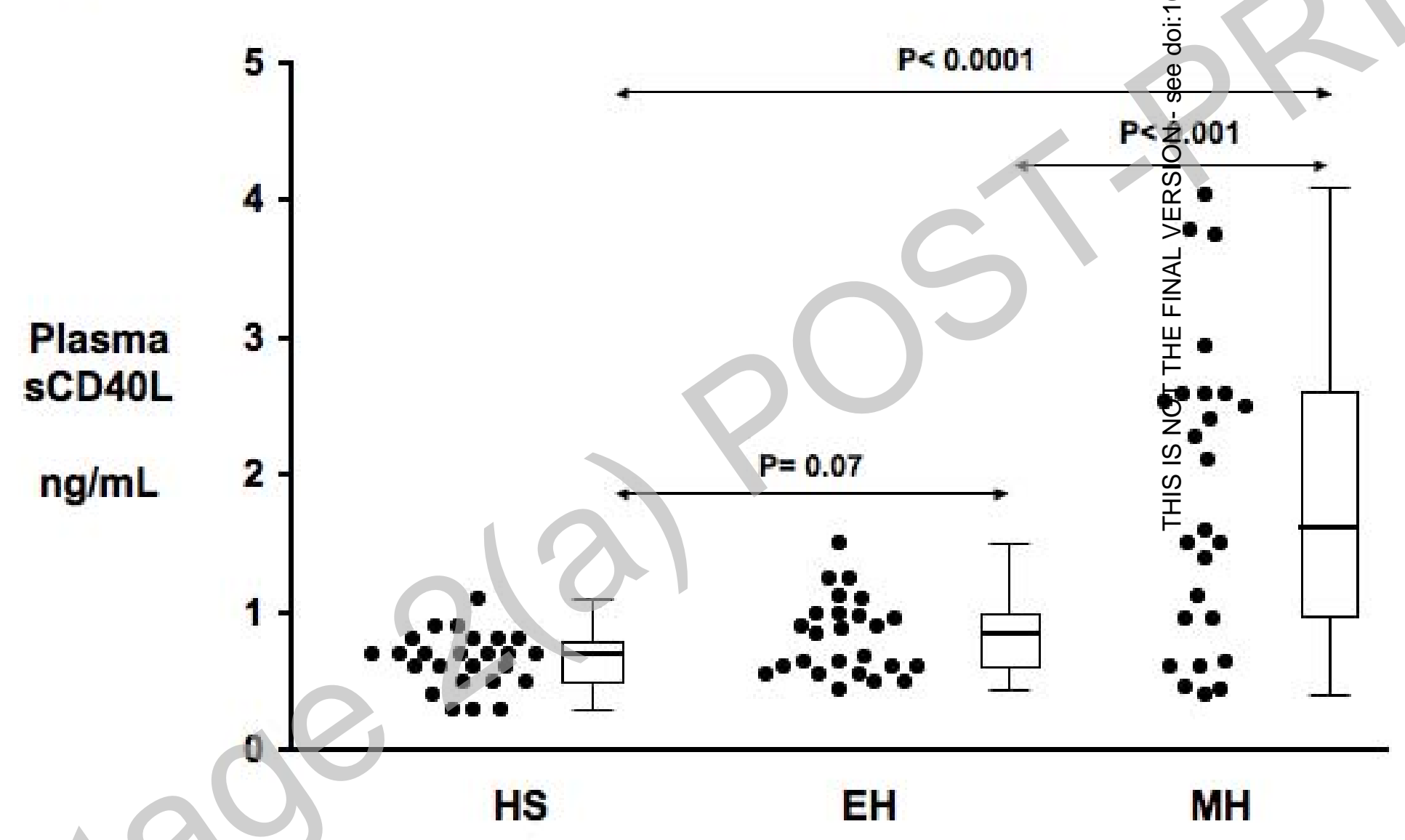


Figure 2
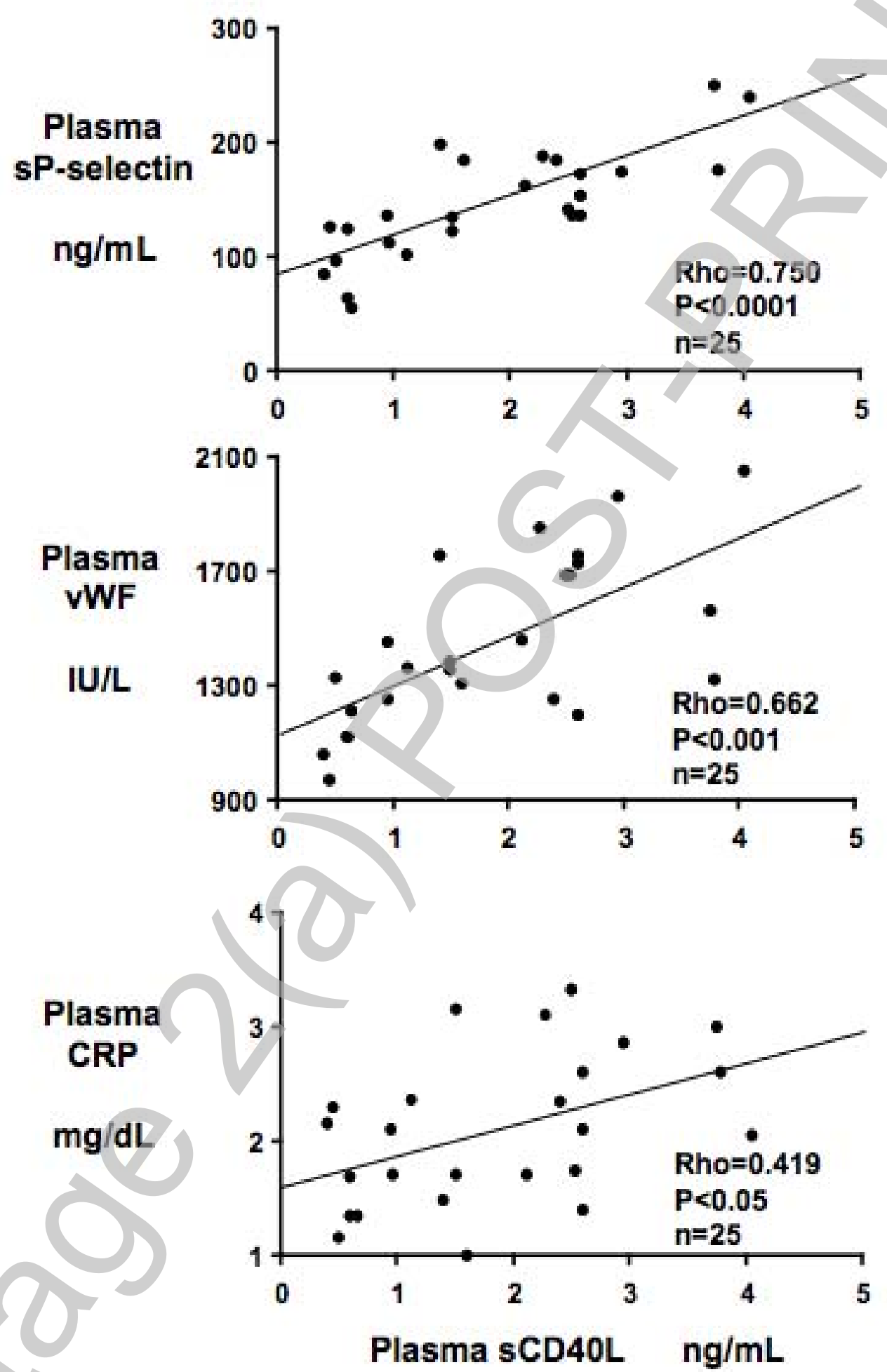

Licenced copy. Copying is not permitted, except with prior permission and as allowed by law. (c) 2007 The Authors Journal compilation (c) 2007 Biochemical Society 
Figure 3

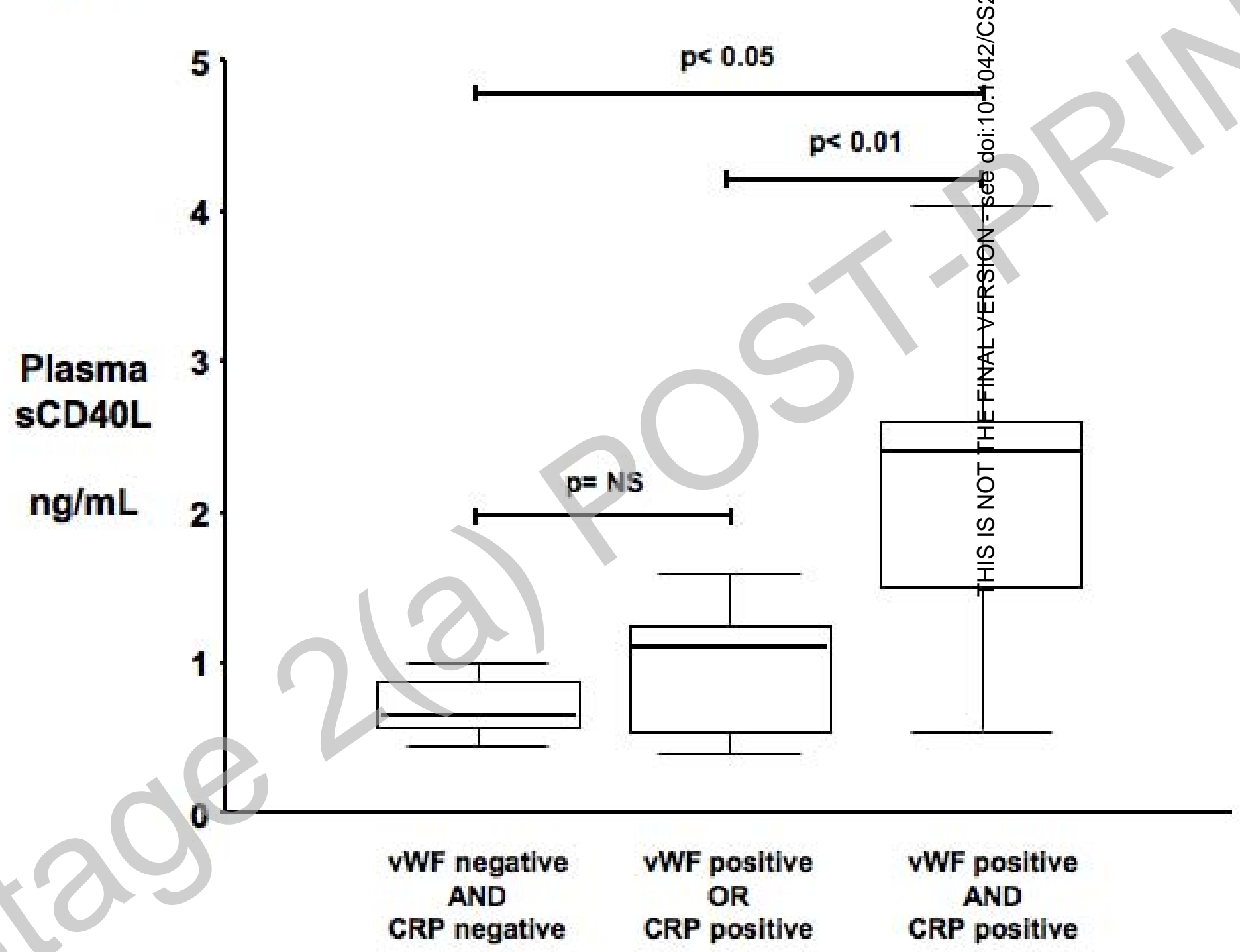

Licenced copy. Copying is not permitted, except with prior permission and as allowed by law. (c) 2007 The Authors Journal compilation (c) 2007 Biochemical Society 
Figure 4

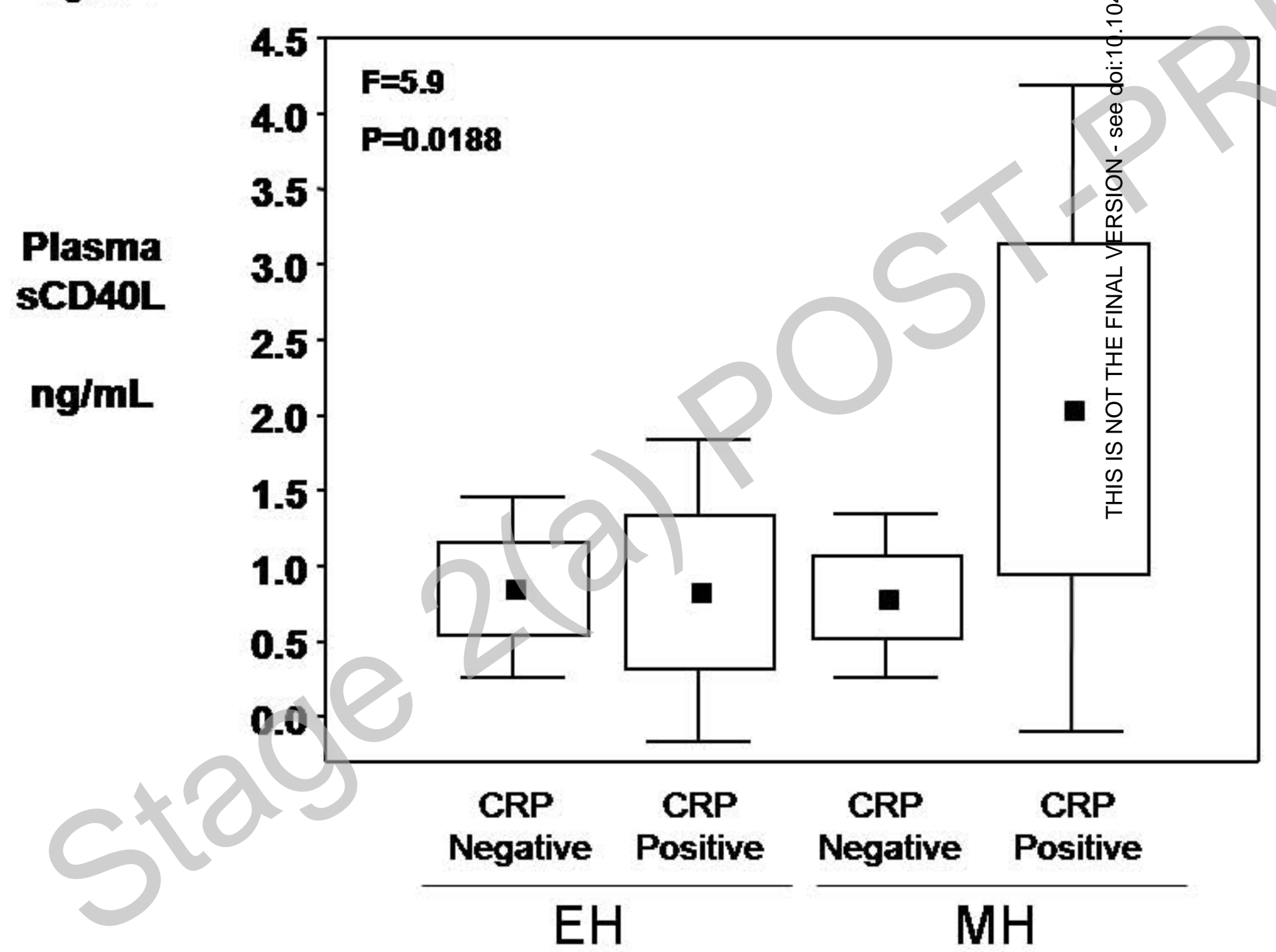

